\title{
3-D PRINTED MICROFLUIDIC DEVICES FOR ELECTROHYDRODYNAMIC GENERATION OF CORE-SHELL MICROPARTICLES
}

\author{
D. Olvera-Trejo ${ }^{1,2}$, and L.F. Velásquez-Garcia ${ }^{2 *}$ \\ ${ }^{1}$ Tecnologico de Monterrey, Monterrey, NL, Mexico \\ ${ }^{2}$ Microsystems Technology Laboratories, Massachusetts Institute of Technology, Cambridge, MA, USA
}

\begin{abstract}
We report the first 3-D printed coaxial electrospray devices for uniform generation of core-shell microparticles; the microencapsulation technology is of great importance in applications such as controlled drug release and self-healing composites. The devices, fabricated with stereolithography, produce core-shell microparticles from coaxial spouts with 150 $\mu \mathrm{m}$-thick walls fed by $700 \mu$ m-diameter microchannels. Experimental characterization of single- and double-emitter devices using deionized water as inner flow and sesame oil as outer flow demonstrate uniform emitter operation; the per-emitter current is proportional to the square root of the flow rate of the driving liquid (water) and is independent of the flow rate of the driven liquid (oil), as predicted by the theory. The average outer diameter of the core-shell microparticles can be modulated by varying the flow rates fed to the spouts.
\end{abstract}

\section{INTRODUCTION}

Coaxial electrospray is an electrohydrodynamic process that creates core-shell microparticles by atomizing a coaxial electrified jet composed of two immiscible liquids [1]. Coaxial electrospray has several advantages over other microencapsulation technologies including higher encapsulation efficiency and more uniform size distribution [2]. In the electrospray phenomenon, a high electrostatic field acting on the free surface of a liquid triggers an instability that transforms the meniscus into a conical shape, called a Taylor cone, that balances the capillary forces and the electrostatic pressure [3]. In the electrospray cone-jet mode, a jet of liquid is ejected from the apex of the cone due to the high electric fields present there; the jet breaks up into droplets. In coaxial electrospray, the liquid fed to the Taylor cone has a coaxial structure composed by an inner liquid and an outer liquid that are immiscible. The same coaxial structure is present in the emitted jet, which generates core-shell droplets when it breaks up: the core of the particle is made of the inner liquid, while the shell of the particle is made of the outer liquid.

The stability of a coaxial Taylor cone and its electrified compound jet strongly depend on the physical properties of the working fluids as well as the supplied flow rates and applied bias voltage. Scaling laws of the dependence of the emitted current and droplet size on the flow rates and the physical properties of the liquid while the emitter is operating in cone-jet mode have been reported [4]. Also, the shell thickness and outer diameter of the core-shell particles can be estimated as a function of the flow rates and the expressions for the inner and outer diameters of the jet [5].

In coaxial electrospray the liquid with the smaller electrical relaxation time is the driving liquid, and the liquid with the larger electrical relaxation time is the driven liquid. A very stable coaxial electrospray configuration entails having the core of the coaxial flow made of the driving liquid, while having the outer layer of the coaxial flow made of the driven liquid; in this case, the electrical stresses acting on the charged inner liquid-outer liquid interface are efficiently transmitted by viscosity to the bulk of the outer liquid, setting it into motion.

Additive manufacturing is a set of free-form fabrication technologies that create solid objects using a computer-aided design (CAD) file as template. Additive manufacturing started as a visualization tool of mesoscaled objects, but recent developments in the resolution and capabilities of 3-D printing suggest that these manufacturing processes could address the complexity, threedimensionality, and material processing capabilities of many microsystems [6]. Stereolithography (SLA) is an additive manufacturing process that creates solid objects from the photopolymerization of a resin using ultraviolet light. Highresolution SLA can be used to manufacture freeform microfluidics at a small fraction of the cost per device, infrastructure cost, and fabrication time of a typical silicon-based microfluidic system [7].

Herein, we report the first 3-D printed microfluidic devices that generate core-shell particles via electrohydrodynamic jetting. Unlike traditional, i.e., uniaxial, electrospray that has been investigated for over 100 years [8] and of which many MEMS implementations exist [9], coaxial electrospray was discovered in 2002 [1] and no microfabricated coaxial electrospray source had been reported due to the inherent three-dimensionality and complexity of its hydraulic system.

\section{DESIGN}

The 3-D printed microfluidic devices, made of an ABS-like material, have one or two $12 \mathrm{~mm}$-tall internally-fed coaxial electrospray emitters that are fed by two $700 \mu \mathrm{m}$-diameter, $37 \mathrm{~mm}$ long helical channels (Figure 1). The channels act as high hydraulic impedances that regulate and uniformize the inner and outer flows fed to the emitter. Each emitter spout is designed to produce a coaxial flow and to enhance the electric field on the liquid meniscus. The emitter tip has a diameter of $2.5 \mathrm{~mm}$ and both the inner and outer outlets of the spout have a hydraulic diameter of $700 \mu \mathrm{m}$. Each emitter occupies a $9.6 \mathrm{~mm}^{2}$ footprint.

Each device has a $7 \mathrm{~mm}$ by $24.25 \mathrm{~mm}$ by $24.25 \mathrm{~mm}$ frame with two liquid reservoirs with a capacity of $250 \mu \mathrm{L}$ each that feed the emitter(s). The frame includes four through-holes that can be used to clamp the device to a chuck with liquid feedthroughs via M4 screws. Between the emitters and the through-holes there are curved spill guards that protect the screws of any spilled liquid.

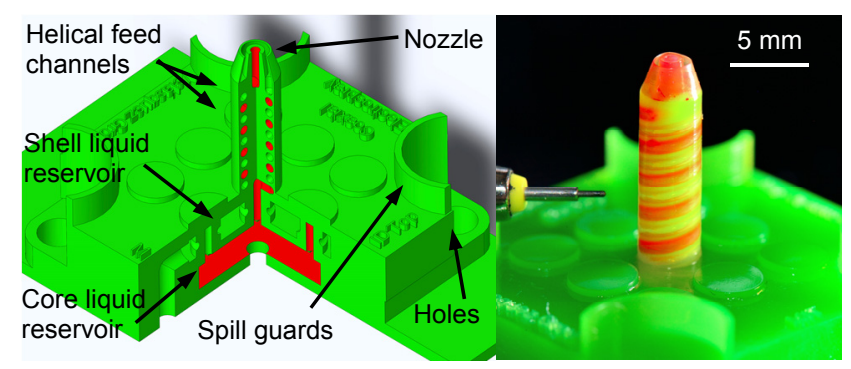

Figure 1: Left: schematic of a single-emitter coaxial electrospray source showing the helical feed channels, liquid reservoirs, and spill guards. Right: photograph of a single-emitter device next to a $0.3 \mathrm{~mm}$-diameter mechanical pencil; the different colors of the liquids supplied to the device evidence the helical channels that feed the emitter nozzle. 


\section{CHARACTERIZATION}

The apparatus shown in Figure 2 was used to characterize the 3-D printed coaxial electrospray sources and to explore how the flow rates and bias voltage influence the stability of the microencapsulation process. Deionized water and sesame oil are loaded into $1 \mathrm{ml}$ syringes controlled by a Dual Syringe 33 Harvard Pump. The chip is clamped to the aluminum chuck using screws and the liquid feedthroughs of the chuck are connected to the syringe pump. The screws are also used to integrate an extractor electrode, i.e., a laser-cut $250 \mu \mathrm{m}$-thick 304 stainless steel plate with apertures that line up with the axes of the coaxial electrospray emitters of the chip. The collector electrode is a $250 \mu \mathrm{m}$-thick 304 stainless steel plate. The aluminum chuck is grounded, a $4.2 \mathrm{kV}$ to $6.4 \mathrm{kV}$ negative bias voltage is applied to both extractor and collector electrodes, using a dual output Gamma High Voltage, 20W, $1 \mathrm{~mA}$ Power Supply. The emitted current was measured with a 485 Keithley picoammeter and recorded by a DS6000 Rigol oscilloscope, the picoammer was connected in series with a large resistor.

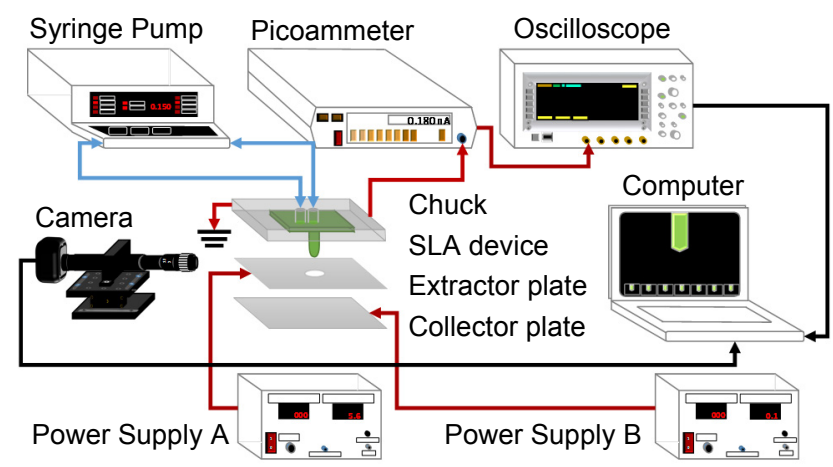

Figure 2: Schematic of experimental apparatus.
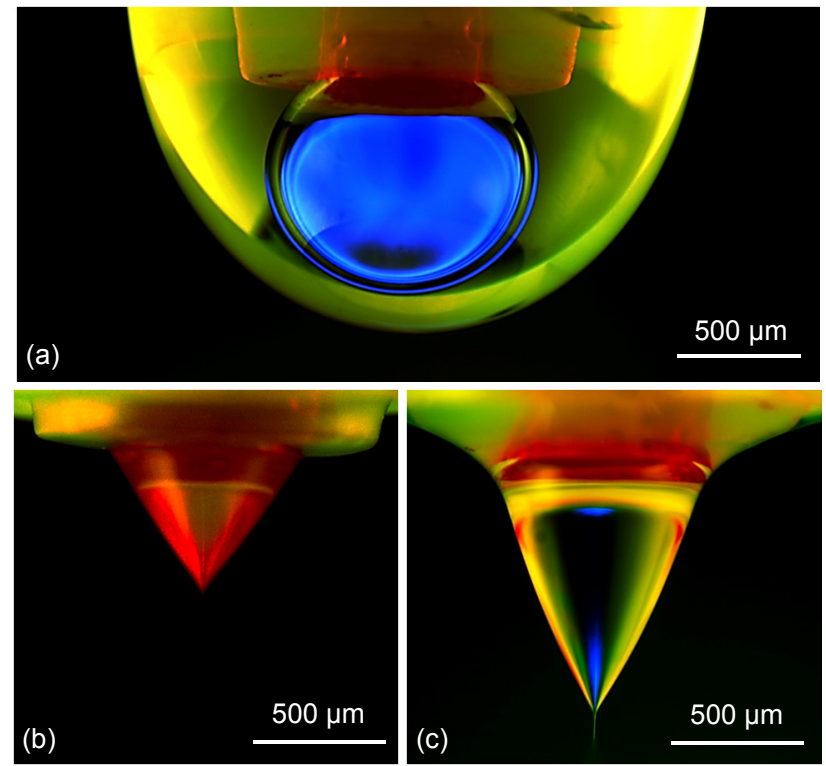

Figure 3: (a): compound droplet of deionized water covered by sesame oil at zero electrical field; (b): uniaxial electrospray of deionized water mixed with Rhodamine $B ;(c)$ : coaxial electrospray of deionized water mixed with Rhodamine $B$ (inner flow) and sesame oil (outer flow). In Figure 3(c) the emitted jet is visible.
Figure 3(a) shows a compound meniscus of deionized water (internal liquid) and sesame oil (external liquid) at the spout of a coaxial electrospray emitter that is not energized. The internal layer of the compound meniscus was illuminated with an ultraviolet lamp shining at an appropriate direction. If the negative bias voltage on the extractor electrode is set to about $5 \mathrm{kV}$, the liquid meniscus triggers electrospray emission in cone-jet mode. In Figure 3(b) only the internal liquid was fed to the chip, resulting in uniaxial electrospray emission, while in Figure 3(c) both the internal liquid and the external liquid were supplied, resulting in coaxial electrospray emission. In both cases, the deionized water was dyed with Rhodamine B to more easily distinguish it from the sesame oil.

\section{Coaxial electrospray modes}

In electrospray different spraying modes can take place according to the magnitude of the electrostatic field and flow rate fed to the emitter. For a given flow rate, increasing the magnitude of the electrostatic field results in operating the emitter in the dripping mode, the cone-jet mode, or the multi-jet mode [10]. In the dripping mode, the bias voltage causes an electrostatic force on the meniscus that is smaller than the hydrodynamic forces, the surface tension forces, and gravity. Therefore, the generation of droplets in this regime is set by the balance between gravity and surface tension (i.e., the droplets fall down if the gravity is large enough to overcome the surface tension), although the droplets still transport charge when a non-zero bias voltage is applied; however, in the dripping mode no core-shell droplets can be generated. In the cone-jet mode the strength of the electric field is large enough to trigger the formation of the Taylor cone, which produces coreshell particles via the break up of the jet emitted by its apex. The cone-jet mode is very stable. Further increasing the bias voltage leads to emission instability; the multi-jet mode appears when more than one jet is emitted from the surface of meniscus. The surface of the meniscus could also become close to the outlet of the emitter, leading to pulsating and unstable jets.

The emitted current was measured to identify the coaxial electrospray modes. To validate this approach, the leakage current through the dielectric was first characterized using a dry chip while the negative bias voltage between the chip chuck and the extractor electrode was set at $5 \mathrm{kV}$. From these measurements, an average leakage current equal to $6 \mathrm{nA}$ was obtained, which is at least twenty times smaller than the current measurements obtained with chips fed by the working liquids.

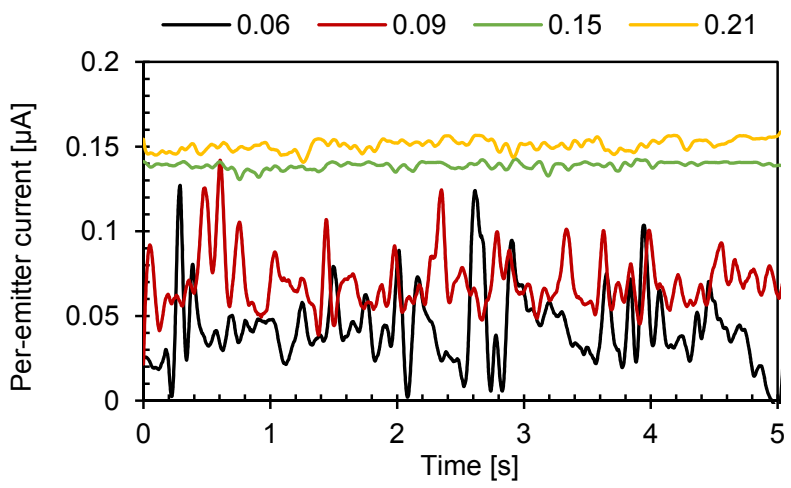

Figure 4: Per-emitter current versus time for deionized water flow rates between $0.06 \mathrm{ml} / \mathrm{hr}$ and $0.21 \mathrm{ml} / \mathrm{hr}$ and a fixed sesame oil flow rate of $0.06 \mathrm{ml} / \mathrm{hr}$. Steady cone-jet emission occurs when the deionized water flow rate is equal or larger than $0.15 \mathrm{ml} / \mathrm{hr}$; below that value, dripping emission occurs. 
In the wet experiments, the extractor bias voltage was set at around $-5 \mathrm{kV}$ and the inner and outer flow rates were varied between $0.06 \mathrm{ml} / \mathrm{hr}$ and $0.30 \mathrm{ml} / \mathrm{hr}$; the results of these experiments are shown in Figure 4. The dripping mode was observed for several flow rate combinations (red and black lines); for instance, when the flow rate of the sesame oil is $0.06 \mathrm{ml} / \mathrm{hr}$, the dripping mode occurs when the water flow rate falls below $0.15 \mathrm{ml} / \mathrm{hr}$. The dripping mode is identified by a seemingly random fluctuation in the emitted current between zero and about $150 \mathrm{nA}$, which is in striking contrast to the steadiness of the emitted current in the cone-jet mode (green and yellow curves). The emitted current in the cone-jet mode is very constant, even after many minutes of continued emission.

When the deionized water and sesame oil flow rates are set at $0.30 \mathrm{ml} / \mathrm{hr}$ and $0.10 \mathrm{ml} / \mathrm{hr}$, respectively, and the extractor electrode is positioned $6.4 \mathrm{~mm}$ from the emitter spout(s), steady cone-jet mode is achieved for extractor bias voltages between -5.4 and -6.3 $\mathrm{kV}$. Similarly, if the plate is positioned $4.5 \mathrm{~mm}$ from the emitter spout(s), a stable Taylor cone can be generated for the wider bias voltage range between $-4.2 \mathrm{kV}$ and $-5.9 \mathrm{kV}$. However, if the extractor electrode is positioned $2.6 \mathrm{~mm}$ from the emitter spout(s), the range of bias voltages that generate a stable Taylor cone is -4.5 to $-4.8 \mathrm{kV}$. It is important to mention that in these experiments the per-emitter current was always very steady and the same $(100 \pm 4$ nA). Therefore, the extractor voltage and position of the extractor electrode seems to have no influence on the per-emitter current; however, the electrical field acting on the Taylor cone increases as the separation distance between the emitter spout and the extractor electrode decreases, which has a significant influence in the formation of the Taylor cone. In addition, for a fixed extractor electrode-to-emitter nozzle separation, increasing the oil flow rate requires to also increase the magnitude of the bias voltage to maintain a stable Taylor cone because the oil affects the electrical fields felt by the driving liquid.

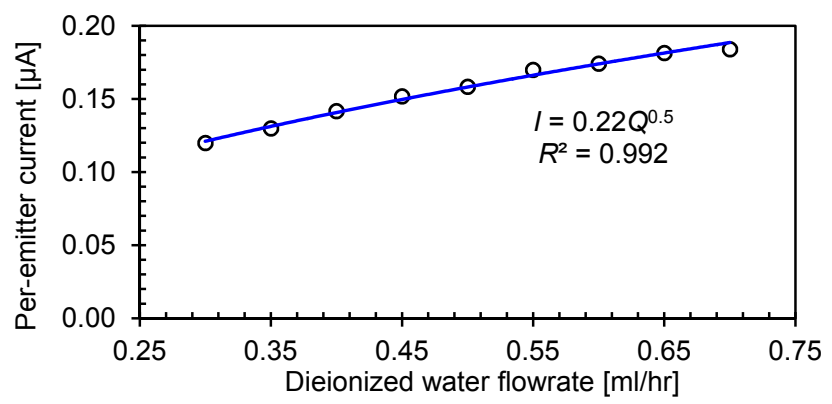

Figure 5: Per-emitter current I versus deionized water flow rate $Q$ when sesame oil's flow rate is $0.10 \mathrm{ml} / \mathrm{hr}$.

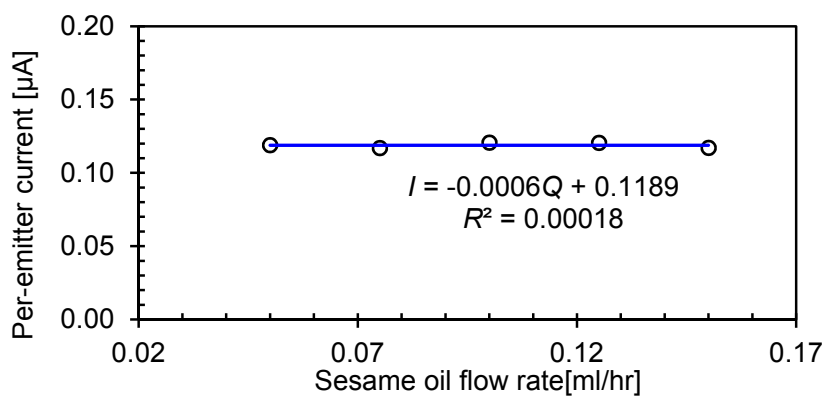

Figure 6: Per-emitter current I versus sesame oil flow rate $Q$ when deionized water's flow rate is $0.30 \mathrm{ml} / \mathrm{hr}$.
Per-emitter current versus flow rate characteristics were also collected to investigate the influence of both flow rates in the formation of a stable coaxial cone-jet. The experiments show that the scaling law that governs standard, i.e., uniaxial, electrospray also applies to the coaxial electrospray. However, in the case of the coaxial electrospray emitter, the flow rate that influences the emitted current is only that of the driving liquid. Steady peremitter currents, which are associated with stable cone-jet formation, are proportional to the square root of the per-emitter flow rate of the driving liquid, -in this case deionized water (Figure 5); this is in agreement with the scaling laws reported in the literature [4]. However, the per-emitter current is not significantly affected by changes in the driven liquid flow rate -in this case sesame oil (Figure 6). The close similarity between the electrical behavior of the uniaxial electrospray and the coaxial electrospray is expected because the oil is very inefficient at transporting charge and for practical purposes it only supplies dielectric material that surrounds the conductive material, similar to operating a standard electrospray emitter in air.

\section{Core-shell particle observation and measurement}

Compound microdroplets were collected on a cover slide placed on top of the collector electrode. The separation distance between the collector electrode and the extractor electrode was varied between $6 \mathrm{~mm}$ and $20 \mathrm{~mm}$, and the collector electrode was biased with voltages between $-1 \mathrm{kV}$ and $-6 \mathrm{kV}$. In order to collect microdroplets, a cover glass was positioned carefully above the collector plate once that electrospray process was running steadily. Then, the collector voltage was turned on for 10 seconds, after which the syringe pump and both power supplies were switched off. After that, the cover glass was observed in an optical microscope. In these experiments the deionized water was dyed with Rhodamine B. After droplet deposition, the cover slide was flooded in water mixed with fluorescein, which greatly helped identify the structure of the compound droplets.

Collection of microdroplets on the slide was observed when the collector electrode-to-extractor electrode separation was at 6 $\mathrm{mm}$ and the collector bias voltage was set at $-5.6 \mathrm{kV}$, i.e., until the magnitude of the bias voltage on the collector electrode is greater or equal to the bias voltage on the extractor plate; this is because the electrical field between the collector and extractor electrodes is close to zero (see Figure 7). In other words, when the collector electrode is biased at a less negative voltage than the extractor electrode, the microdroplets decelerate, which leads to collecting the microdroplets on other surfaces.

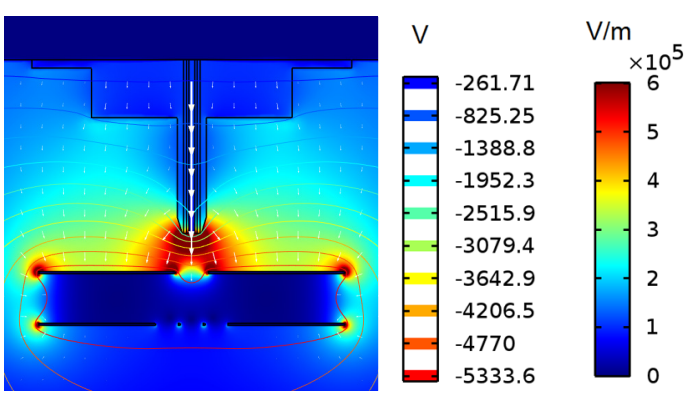

Figure 7: Axisymmetric finite element simulation of the electrical field (magnitude is shown) and electrical potential (contour lines) for a coaxial electrospray emitter. The chuck was grounded, both extractor and collector bias voltage were set at $-5.6 \mathrm{kV}$. These conditions correspond to those of the experiment used for droplet collection. 


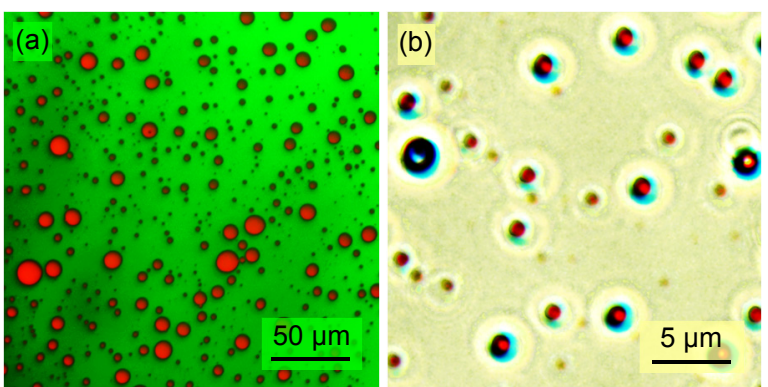

Figure 8: (a): fluorescent image of core-shell microdroplets (red) flooded in water with fluorescein (green); (b): close-up of microdroplets with core made of deionized water with Rhodamine $B$ coated by a sesame oil shell.

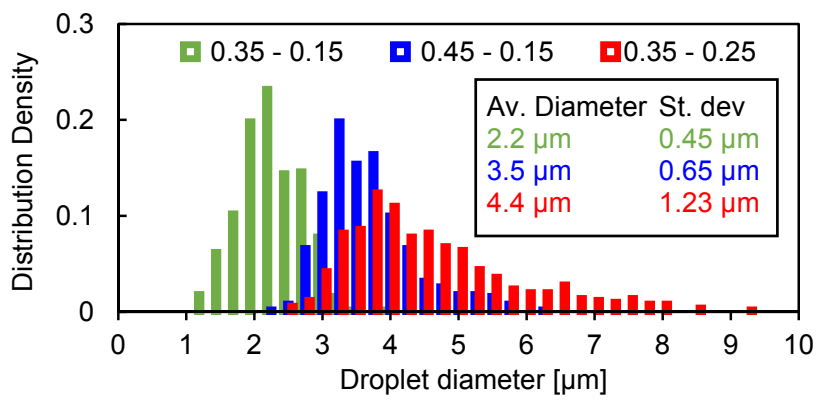

Figure 9: Statistical distribution of the outer diameter of the coreshell particles for different deionized water mixed with Rhodamine $B$-olive oil flow rates $[\mathrm{ml} / \mathrm{hr}]$.

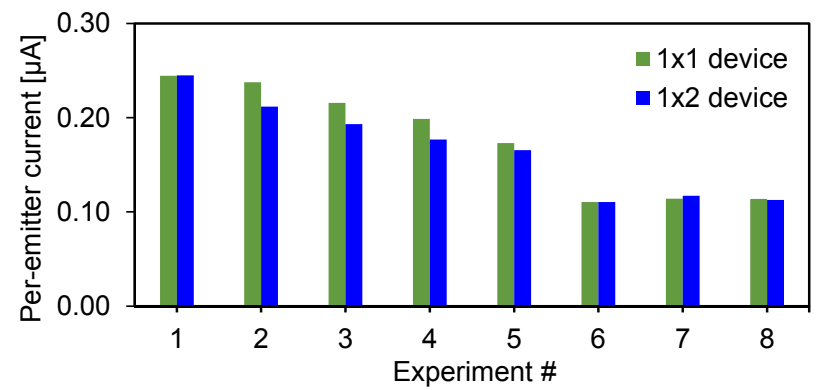

Figure 10: Per-emitter current from single- (green) and doubleemitter (blue) 3-D printed coaxial electrospray sources for various flow rates. Experiments 1, 2, 3, 4 and 5: sesame oil flow rate = $0.50 \mathrm{ml} / \mathrm{hr}$, deionized water flow rate $=2.0,1.75,1.50,1.25$ and $1.00 \mathrm{ml} / \mathrm{hr}$, respectively. Experiments 6, 7, and 8: deionized water flow rate $=0.40 \mathrm{ml} / \mathrm{hr}$, sesame oil flow rate $=0.20,0.15$, and 0.10 $\mathrm{ml} / \mathrm{hr}$, respectively. The double-emitter devices are assumed to operate uniformly, i.e., the per-emitter current and flow rate are equal to diving the total current and total flow rate by two.

The core-shell structure of the droplets collected on the slide is visible using fluorescent microscopy, as shown in Figure 8. In Figure 8(a), red microdroplets can be seen in a green background because the red-dyed cores of the particles are encapsulated in sesame oil, which refrains their mixing with the water dyed with fluorescein. In Figure 8(b), the red core of the compound droplets, as well as the translucent oil shell, are visible.

The diameter distribution of compound microdroplets produced with different combinations of driving liquid and driven liquid flow rates was characterized using metrology of images from an optical microscope (Figure 9). In each case, 500 droplets were measured to estimate the average and standard deviation of the outer droplet diameter. The experimental data demonstrate that the droplets' average outer diameter and standard deviation can be modulated by controlling the flow rates. In particular, if the flow rate of the driving liquid is significantly larger than that of the driven fluid, the distribution has associated a smaller standard deviation. In addition, smaller flow rates result in smaller compound droplets.

Figure 10 shows the per-emitter current from single- and double-emitter sources under various flow rate conditions; the results suggest that the emitters are operating uniformly.

\section{CONCLUSION}

We reported the first 3-D printed coaxial electrospray devices for generation of core-shell microparticles. Characterization of single- and double-emitter devices using deionized water as inner flow and sesame oil as outer flow demonstrate uniform emitter operation and generation of core-shell microparticles with narrow diameter size distribution. Current research efforts focus on achieving high-throughput core-shell microparticle generation through emitter multiplexing [7] combined with integration of electrodes via MEMS assembly technologies [11].

\section{ACKNOWLEDGEMENTS}

This research has been partially funded by the Tecnológico de Monterrey and MIT Nanotechnology Program.

\section{REFERENCES}

[1] I.G. Loscertales et al, "Micro/nano encapsulation via electrified coaxial liquid jets", Science, 295, 1695 (2002).

[2] L. Zhang, J. Huang, T. Si, and R. X Xu, "Coaxial electrospray of microparticles and for biomedical applications", Expert Rev. Med. Devices, 9, 595 (2012).

[3] I.G. Taylor, "Disintegration of water drops in an electric field", Royal Society of London A, 280, 383 (1964).

[4] J.M. López-Herrera, A. Barrero, A. López, I.G. Loscartales, M. Márquez, "Coaxial jets generated from electrified Taylor cones. Scaling laws", J. Aerosol Science, 34, 535 (2003).

[5] A.G. Marín, I.G. Loscertales, M. Márquez and A. Barrero, "Simple and Double Emulsion via Coaxial Jet Electrosprays", Physical Review Letters, 98 (2007).

[6] K.V. Wong and A. Hernandez, "A review of additive manufacturing”, ISRN Mechanical Engineering, 2012.

[7] L.F. Velásquez-García, "SLA 3-D printed arrays of miniaturized, internally fed, polymer electrospray emitters", J. Microelectromech. Systems, 24, 2117 (2015).

[8] J. Zeleny, "The electrical discharge from liquid points, and a hydrostatic method of measuring the electric intensity at their surfaces", Physical Review, 3, 69 (1914).

[9] L.F. Velásquez-García, A.I. Akinwande, and M. MartínezSánchez, "A micro-fabricated linear array of electrospray emitters for thruster applications", J. Microelectromech. Systems, 15, 1260 (2006).

[10] N. Bock, T.R. Dargaville, "Electrospraying of polymers with therapeuthic molecules: State of the art", Progress in Polymer Science, 37, 1510 (2012).

[11] B. Gassend, L.F. Velásquez-García, and A.I. Akinwande, "Precision in-plane hand assembly of bulk-microfabricated components for high-voltage MEMS arrays applications", J. Microelectromech. Systems, 18, 332 (2009).

\section{CONTACT}

*L.F. Velásquez-Garcia, tel: +1-617-253-0723; 1fvelasq@mit.edu 